\title{
超音速激光沉积 $\mathrm{WC} / \mathrm{Cu}$ 复合涂层的微观结构及 耐磨性能表征 ${ }^{*}$
}

\author{
黄煊杰 ${ }^{1,2}$ 吴丽娟 ${ }^{1,2}$ 李 波 ${ }^{1,2}$ 汪伟林 ${ }^{1,2}$ 张群莉 1,2 \\ 弗拉基米尔・科瓦连科 1,3 姚建华 1,2 \\ (1. 浙江工业大学激光先进制造研究院 杭州 310014; \\ 2. 浙江省高端激光制造装备协同创新中心 杭州 310014; \\ 3. 乌克兰国立科技大学激光技术研究所 基辅 03056 乌克兰)
}

摘要: 针对纯铜具有良好的导电、导热和加工性能, 但同时也具有硬度低, 耐磨性能差的特点。采用超音速激光沉积(Supersonic laser deposition, SLD)和冷喷涂(Cold spray, CS)技术在纯铜表面制备了 $\mathrm{WC} / \mathrm{Cu}$ 复合涂层, 并对所制备涂层的微观结构、相组 成、显微硬度和摩擦磨损性能进行了对比分析。研究结果表明, CS 涂层的厚度约为 $1128 \mu \mathrm{m}, \mathrm{WC}$ 含量为 $7.73 \%$, 显微硬 度为 $147.4 \mathrm{HV}_{0.2}$, 但涂层/基体结合处存在明显间隙。 SLD 涂层厚度随着激光功率的逐渐升高而增加, 最高涂层厚度达 2 $344 \mu \mathrm{m}, \quad \mathrm{WC}$ 含量可高达 $17.22 \%$, 显微硬度可达 $161.3 \mathrm{HV}_{0.2}$, 且涂层/基体结合良好。SLD 涂层能基本保留原始粉末的相 组成, 但高激光功率下制备的样品存在轻微氧化。SLD 涂层相比于 CS 涂层和铜基体具有更小的摩擦因数、磨损宽度以及磨 损量, 表现出更好的耐磨性能, 为铜及其合金的表面性能优化提供了一种新方法。

关键词: 超音速激光沉积; $\mathrm{WC} / \mathrm{Cu}$ 复合涂层; 固态沉积; 微观结构; 显微硬度; 耐磨损性能 中图分类号: TG146

\section{Microstructure Characterization and Tribological Properties Evaluation on WC/Cu Composite Coating Prepared by Supersonic Laser Deposition}

\author{
HUANG Xuanjie WU Lijuan $^{1,2}$ LI Bo ${ }^{1,2}$ WANG Weilin ${ }^{1,2}$ ZHANG Qunli ${ }^{1,2}$ \\ VOLODYMYR S. Kovalenko ${ }^{1,3}$ YAO Jianhua ${ }^{1,2}$ \\ (1. Institute of Laser Advanced Manufacturing, Zhejiang University of Technology, Hangzhou 310014; \\ 2. Zhejiang Provincial Collaborative Innovation Center of High-end Laser Manufacturing Equipment, \\ Hangzhou 310014; \\ 3. Laser Technology Research Institute, National Technical University of Ukraine, Kiev 03056 Ukraine)
}

\begin{abstract}
Pure copper has good conductivity of heat and electricity, but low hardness and poor wear resistance. Therefore, the WC/Cu composite coatings are prepared on the surface of copper substrate by supersonic laser deposition (SLD) and cold spray (CS). The microstructure, phase composition, microhardness and wear property of the as-prepared coatings are comparatively analyzed for these two technologies. The results show that the thickness of CS coating is about $1128 \mu \mathrm{m}$, the WC content is $7.73 \%$ and the microhardness is 147.4 $\mathrm{HV}_{0.2}$, but there are some gaps at the coating/substrate bonding zone. For SLD coating, the thickness increases with elevating laser power. The thickest coating thickness is up to $2344 \mu \mathrm{m}$, the highest WC content is $17.22 \%$ and the highest microhardness reaches 161.3 $\mathrm{HV}_{0.2}$. All SLD coatings show well-bonded coating/substrate interface. The SLD coating still retains the original phase composition of the feedstock powder. However, samples prepared at high laser power experienced slight oxidation. The wear resistance of the SLD coating is superior to that of the CS coating and $\mathrm{Cu}$ substrate, with lower coefficient of friction, wear scar width and wear loss. These results provide a new route for improving the surface properties of $\mathrm{Cu}$ and its alloys.
\end{abstract}

Key words: supersonic laser deposition; WC/Cu composite coatings; solid-state deposition; microstructure; microhardness; wear-resistance

* 国家自然科学基金(51701182)、国家重点研发计划(2017YFB1103601)、

中国博士后基金面上(2019M662103)和浙江省基础公益研究计划

(LGG19E050024)资助项目。20190605 收到初稿, 20200220 收到修改稿 


\section{0 前言}

铜 $(\mathrm{Cu})$ 及铜合金材料因具有良好的导电、导热 和加工性能, 被广泛应用于航天航空、交通运输和 电子电气等领域的焊接电极、电子封装材料、集成 引线框架、仪器仪表及电子通信设备中的接触元件 等 ${ }^{[1-2]}$ 。然而, 较低的强度、硬度以及较差的耐磨性 能, 限制了其在工业和军事领域的应用 ${ }^{[3-4]}$ 。随着表 面技术的发展, 通过强化服役表面来提升装备运行 的可靠性和延长装备寿命具有显著的经济价值和重 要的社会意义 ${ }^{[5]}$, 因此在铜材料表面制备铜基复合 涂层是在保证其良好性能同时改善其不足的重要趋 势。碳化铇(WC)具有硬度高、熔点高、热膨胀系数 小、耐磨性好等特点, 而且与铜材料的润湿性较好, 因此将其作为铜基复合涂层的增强相, 既能增强复 合涂层表面硬度与耐磨性能, 又能保持较好的导热 性与导电性, 是一种较为理想的选择。

目前研究较多的表面制备方法包括: 电镀法、激 光熔覆法、铸渗法、热喷涂法等。每种方法有其特色, 但也都存在一定的不足。例如, 电镀法获得的涂层与 基体之间为机械结合，非冶金结合，长期使用镀层容 易开裂脱落; 铜对激光能量的反射率高, 导热速度快, 极大限制了激光熔覆在铜基表面复合涂层制备的应 用; 铸渗法容易产生高温有害脆性相, 影响颗粒与基 体的界面结合, 严重时还可能出现孔洞等铸造缺陷; 热喷涂涂层厚度低、内部孔隙率高、与基体结合强度 较低, 且加工温度高, 铜易氧化 ${ }^{[6-10]}$ 。

超音速激光沉积(Supersonic laser deposition, SLD) 是将激光的辐照软化与冷喷涂的绝热剪切失 稳相耦合的一种新的表面改性技术 ${ }^{[11-13]}$ 。该技术采 用激光作为辅助热源, 沉积过程中激光辐照光斑和 喷涂粉斑同步，实现对喷涂颗粒、基材同步加热， 从而使两者得到有效软化, 增强颗粒的变形能力, 大大降低颗粒所需的临界沉积速度。由于颗粒临界 沉积速度的降低, 不仅可以用氮气来替代价格高昂 的氦气使得使用成本降低, 而且克服了原先单一冷 喷涂无法或难以沉积高硬度材料的问题, 显著拓展 了可沉积材料的范围, 同时提高了涂层的沉积效率、 致密度及结合强度等 ${ }^{[14]}$ 。KULMALA 等 ${ }^{[15]}$ 分别利用 低压冷喷涂(Low pressure cold spray, LPCS)和 SLD 在预喷砂的低碳钢 Fe52 上沉积了 $50 \%$ 体积分数 $\mathrm{Al}_{2} \mathrm{O}_{3} / \mathrm{Cu}$ 涂层, 结果表明激光辐照的引入改善了涂 层的致密度和沉积效率; LI 等 ${ }^{[16]}$ 分别利用 CS 和 SLD 在低碳钢表面制备了 $\mathrm{Cu}$ 涂层, 结果表明 SLD
涂层比 CS 涂层表面更光滑, 峰值涂层厚度增加约 $70 \%$ 且涂层和基体结合更好; 骆芳等 ${ }^{[17]}$ 利用模拟所得出 的沉积速度与沉积温度在 45 钢表面制备了 $\mathrm{Cu}$ 涂层, 验证了模拟结果的正确性; 吴丽娟等 ${ }^{[18]}$ 在铜表面沉积 了金刚石/ $\mathrm{Cu}$ 复合涂层, 结果表明原始粉末配比的增加 不能显著提升金刚石含量，涂层的热导率随着金刚石 颗粒含量的增加而降低，随着颗粒粒径的增大而提高。 小粒径金刚石颗粒表现出更优的耐磨损性能。

基于超音速激光沉积的特点与优势，本文采用 超音速激光沉积技术在纯铜基材表面制备 $\mathrm{WC} / \mathrm{Cu}$ 复合涂层, 重点对比超音速激光沉积和冷喷涂技术 在复合涂层的微观结构、成分以及磨损性能的优劣。

\section{1 试验材料和方法}

\section{1 试验设备}

试验所采用的 SLD 系统主要包括光纤耦合半 导体激光器、冷喷涂设备、高压气体供应装置和温 度反馈系统, 如图 1 所示。高压氮气被分为两部分: 一部分用于输送喷涂粉末, 另一部分直接进入喷嘴, 两部分气体在混合腔内重新汇合在一起。粉末颗粒 在 Laval 喷嘴中被高速气流加速到超音速后，喷射 到基体，经过剧烈塑性变形形成涂层。

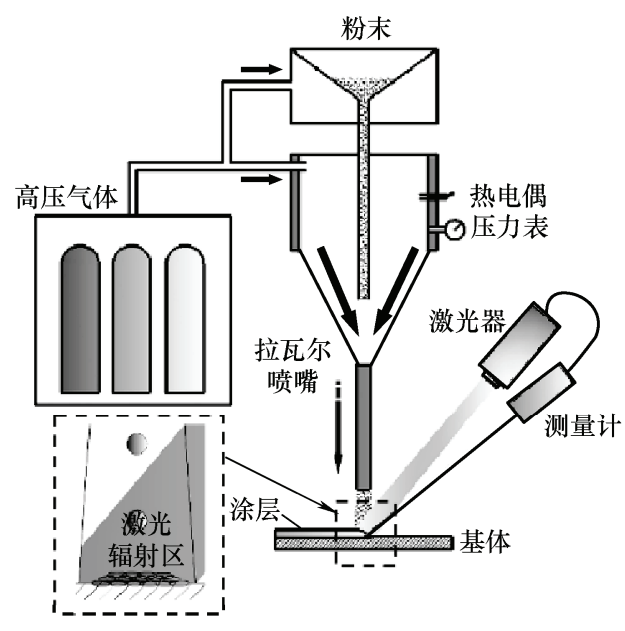

图 1 超音速激光沉积系统示意图

\section{2 试验材料}

沉积粉末材料为纯铜粉与碳化钨的复合粉末, 其中碳化铇的质量分数为 $70 \%$ (体积分数约为 $57 \%$ ), 试验前用球磨法混合。基体材料为 $\mathrm{T} 1$ 紫铜, 试样 尺寸为 $100 \mathrm{~mm} \times 50 \mathrm{~mm} \times 5 \mathrm{~mm}$ 。

\section{3 试验方案}

采用图 1 所示的 SLD 系统制备 $\mathrm{WC} / \mathrm{Cu}$ 复合涂 层, 工艺参数如表 1 所示。利用光学显微镜和扫描 电子显微镜分析沉积层横截面的微观结构和涂层摩 擦磨损后的磨痕。利用 $\mathrm{X}$ 射线衍射仪 (X-ray 
diffraction, XRD)和能谱仪(Energy dispersive X-ray spectroscopy, EDS)对涂层的化学成分进行分析。利 用图像分析软件 Image J 测定复合涂层中 WC 颗粒 的含量。采用 HT-1000 球盘式摩擦磨损仪测试涂层 的摩擦磨损性能。

\section{表 1 超音速激光沉积 $\mathrm{WC} / \mathrm{Cu}$ 复合涂层的工艺参数}

\begin{tabular}{ccccc}
\hline 样品 & $\begin{array}{c}\text { 载气压力/ } \\
\mathrm{MPa}\end{array}$ & $\begin{array}{c}\text { 载气温度/ } \\
{ }^{\mathrm{C}}\end{array}$ & $\begin{array}{c}\text { 激光功率/ } \\
\mathrm{kW}\end{array}$ & $\begin{array}{c}\text { 扫描速度/ } \\
\left(\mathrm{mm} \cdot \mathrm{s}^{-1}\right)\end{array}$ \\
\hline $\mathrm{a}$ & 3 & 450 & 0 & 10 \\
$\mathrm{~b}$ & 3 & 450 & 1 & 10 \\
$\mathrm{c}$ & 3 & 450 & 2 & 10 \\
$\mathrm{~d}$ & 3 & 450 & 3 & 10 \\
\hline
\end{tabular}

\section{2 分析与讨论}

\section{1 复合涂层宏观形貌}

图 $2 a$ 和图 $2 b$ 分别为 $C S$ 和 SLD 制备的 $W C / C u$ 复合涂层横截面, 可以发现, 所有沉积的 $\mathrm{WC} / \mathrm{Cu}$ 复合涂层在宏观上是连续且致密的, 没有明显的裂 缝和孔隙。采用 SEM 的图像分析软件测量每个样品 的涂层厚度, 可用作定性表征沉积效率的指标。从 图 3 中可以看出, $\mathrm{CS}$ 沉积的 $\mathrm{WC} / \mathrm{Cu}$ 复合涂层(样品 a)的涂层厚度约为 $1128 \mu \mathrm{m}$ 。在激光辐照的辅助下, 涂层厚度显著增加, 且随着激光功率从 $1 \mathrm{~kW}$ 逐渐 升高至 $3 \mathrm{~kW}$, 涂层厚度依次为 $1773 \mu \mathrm{m}$ (样品 $\mathrm{b}$ )、

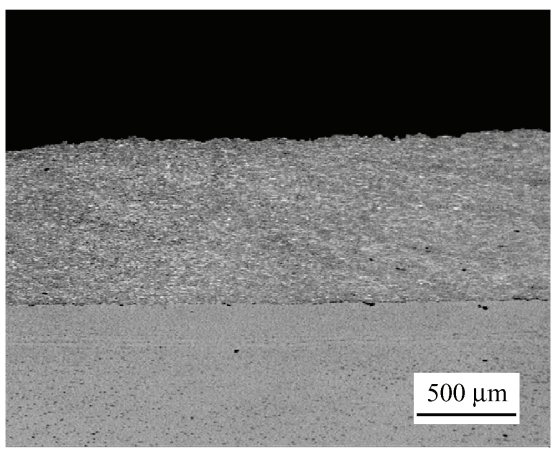

(a) $\mathrm{CS}$

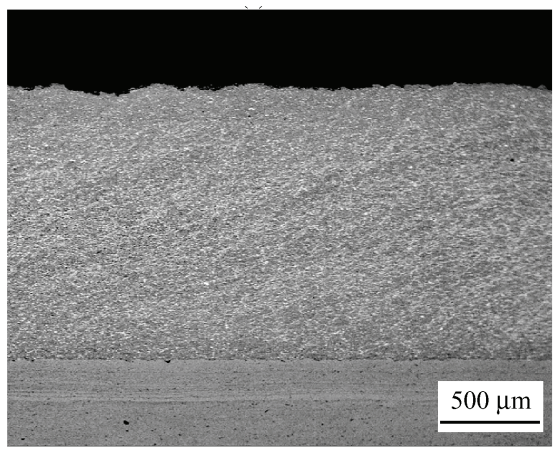

(b) SLD

图 $2 \mathrm{CS}$ 和 SLD 复合涂层截面

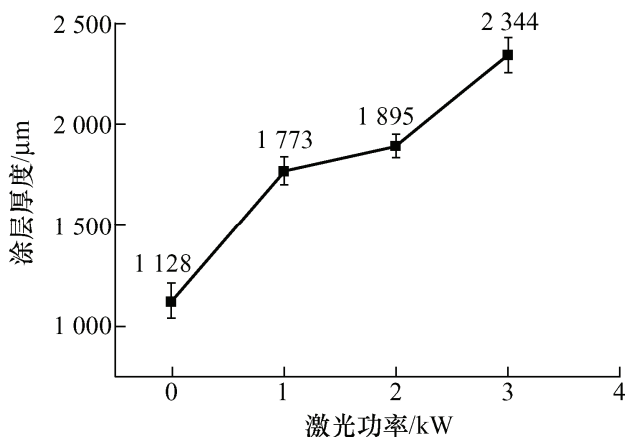

图 3 复合涂层厚度随激光功率的变化规律

$1895 \mu \mathrm{m}$ (样品 $\mathrm{c}$ )和 $2344 \mu \mathrm{m}$ (样品 $\mathrm{d}$ )。在该研究中, 所有 SLD 涂层具有比 CS 涂层更高的涂层厚度, 特 别是当激光功率上升至 $3 \mathrm{~kW}$ 时, 其涂层厚度约是 单一冷喷涂涂层厚度的 2.07 倍。在冷喷涂过程中, 只有当沉积颗粒撞击速度等于或大于其临界沉积速 度时, 才能实现粉末的有效沉积。冷喷涂粉末临界 沉积速度的经验公式如下所示 ${ }^{[19]}$

$$
V_{\mathrm{cr}}=667-14 \rho+0.08 T_{\mathrm{m}}+0.1 \sigma_{\mathrm{u}}-0.4 T_{\mathrm{i}}
$$

式中, $\rho$ 为粉末密度; $T_{\mathrm{m}}$ 为粉末的熔化温度; $\sigma_{\mathrm{u}}$ 为 粉末极限强度; $T_{\mathrm{i}}$ 为粉末撞击基体前的初始温度。

可以看出, 粉末初始温度的升高可以有效地降 低粉末沉积的临界速度。在超音速激光沉积过程中， 由于激光辐照对于粉末与基体的同步加热, 有效地 提升了粉末与基体的初始温度，从而降低了粉末的 临界速度。在本研究中, 对于所有样品, CS 参数 连同使用的喷嘴和原料材料均保持不变, 如表 1 所示, 这表明所有样品在沉积时具有相同的颗粒 撞击速度。因此, 随着激光功率的增加, 粉末的 软化程度以及初始温度都随之增加, 粉末的临界 沉积速度相应减小，撞击速度超过临界速度的粉 末颗粒也增多，从而增加了粉末的沉积效率。这 与我们之前的研究如金刚石/Ni60、Cu、Stellite 6、 $\mathrm{WC} / \mathrm{SS} 316 \mathrm{~L}$ 等 ${ }^{[12,16,20-21]}$ 所观察到的一致。

\section{2 复合涂层界面结合}

图 4 显示了 CS 和 SLD 涂层与基体的界面结合 情况。可以看出, 在 CS 涂层和基体之间存在由于 结合不良而出现的明显缝隙, SLD 涂层的界面结合 良好无缝隙，涂层/基体结合界面处发生材料间的塑 性变形和卷曲混合, 复合涂层与基体结合界面呈机 械咬合机制。在 CS 工艺中，基体的硬度是影响界 面结合强度的重要因素。越柔软的基体越能使沉积 颗粒更深地渗透到其中，促进颗粒和基体表面上的 绝热剪切失稳效应(Adiabatic shear instability, ASI) 的发生以形成更强的结合。在 SLD 工艺中, 激光不 仅预热喷涂颗粒，而且还使基体软化，与 CS 涂层 
相比, 软化的基体将有益于 SLD 涂层形成更好的界 面结合。基体温度的升高对界面结合的有益影响也 得到了其他学者的数值模拟验证 ${ }^{[22]}$ 。

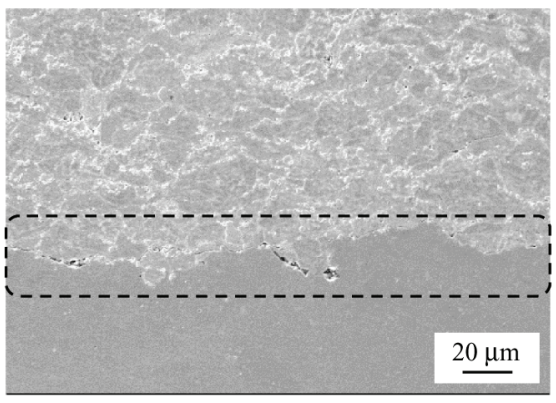

(a) CS

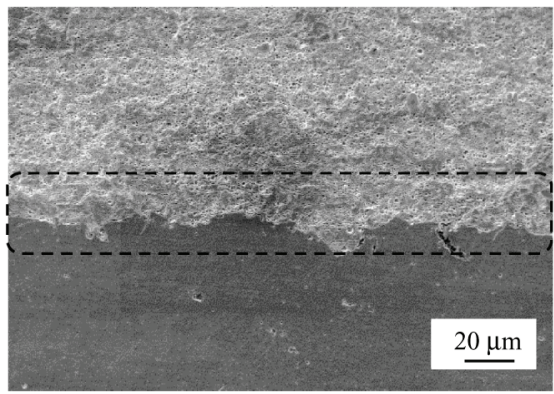

(b) SLD-1 kW

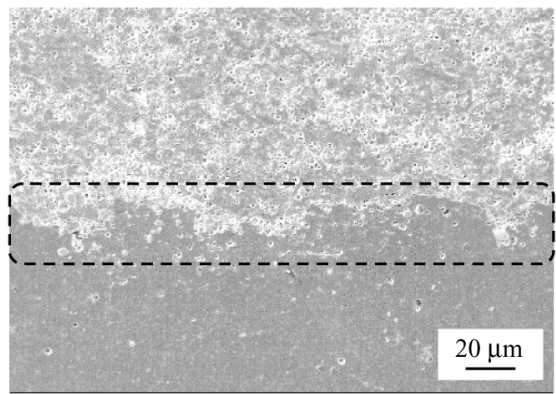

(c) SLD-2 kW

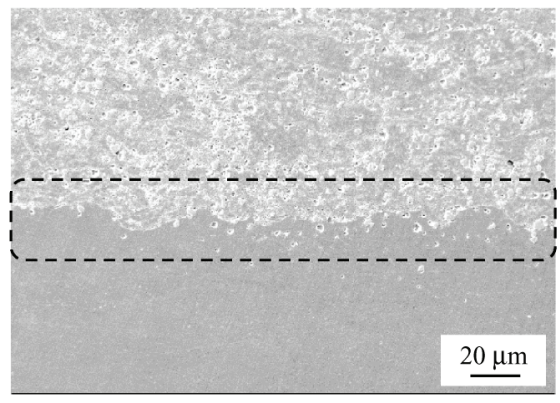

(d) SLD-3 kW

图 4 不同激光功率下的涂层/基体结合区域

\section{3 复合涂层物相分析}

在 SLD 工艺中, 引入了额外的激光辐射热源。 因此有必要研究激光引入对相组成的影响。图 5 是 原始粉末和 SLD 涂层的 XRD 图谱。可以发现, SLD 涂层具有与原始粉末几乎一致的物相, 这证明 SLD 中虽然有激光的引入，但没有引起材料的分解、相 变等, 依旧保留了 $\mathrm{CS}$ 的固态沉积的特性。在基于 激光熔化的金属沉积方法中, 通常采用高激光功率

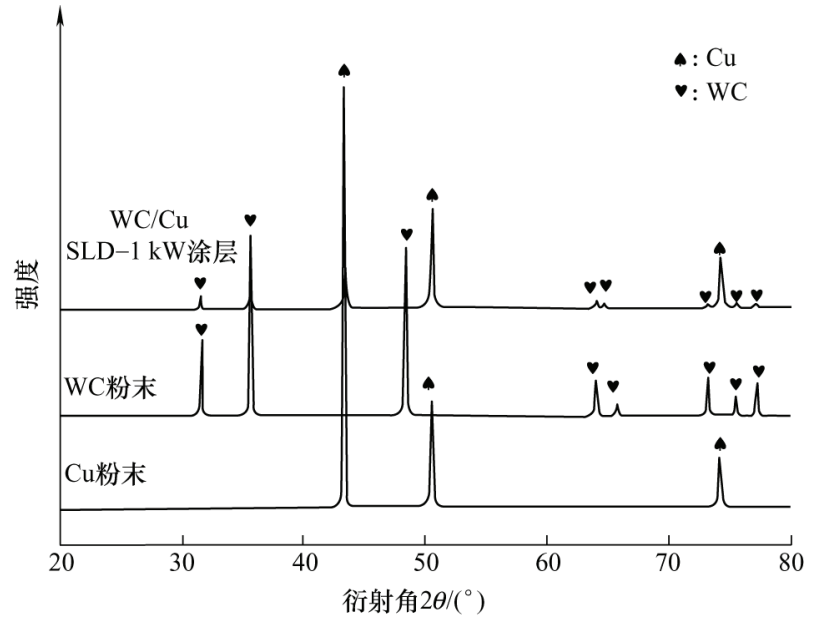

图 5 原始粉末与 SLD 复合涂层的 XRD 图谱

和低扫描速度来获得熔化沉积的粉末和基体所需的 高热输入, 该过程通常会使材料发生相变、分解等 不利影响, 特别是对于诸如金刚石、WC 等热敏材 料。而在 SLD 工艺中, 激光所起的作用是预热沉积 的颗粒与基体，仅仅是软化而不是熔化它们。因此, SLD 是相对低的热输入的过程, 依此来保持原料材 料的原始微观结构和相组成。

对 SLD-1 kW、SLD-2 kW 和 SLD-3 $\mathrm{kW}$ 涂层 进行进一步的 EDS 分析, 结果如图 6 所示。可以发 现, SLD-1 kW 涂层并没有 $\mathrm{O}$ 元素的出现, 而随着 激光功率逐渐增加, SLD-2 $\mathrm{kW}$ 和 SLD-3 $\mathrm{kW}$ 涂层 都检测到不同程度的氧元素, 原子量百分比分别为 2.41 和 3.67。SLD 涂层中 $\mathrm{O}$ 元素的增加应归因于引入激光 辐照时 $\mathrm{Cu}$ 颗粒与周围空气的反应。一方面引入激光可 以有效地软化沉积的颗粒, 以提高它们的塑性变形能 力, 有利于材料的沉积。另一方面, 增加激光功率会 提高 $\mathrm{Cu}$ 氧化的风险, 而生成的 $\mathrm{CuO}$ 属于陶瓷相, 较 高的硬度会在一定程度上影响粉末的沉积效率。因此, 在 SLD 过程中，激光功率不宜太高。

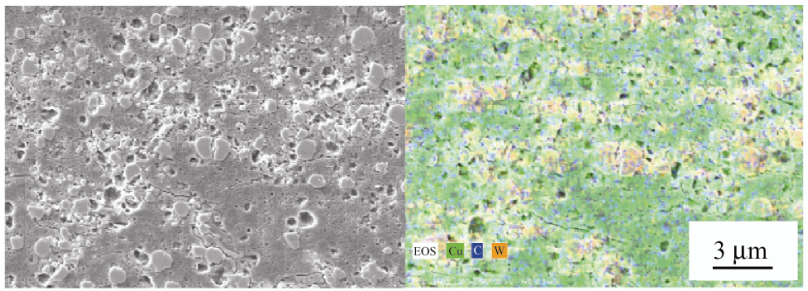

(a) SLD-1 kW

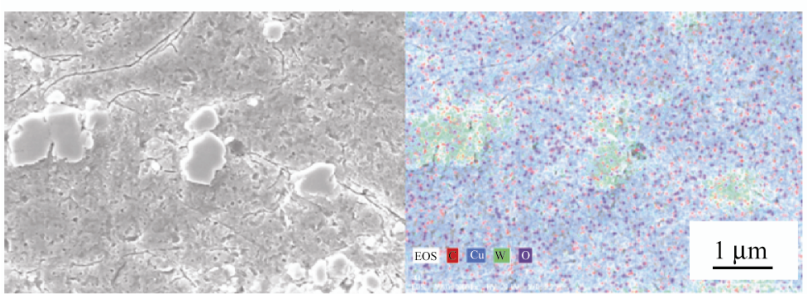

(b) SLD-2 kW 


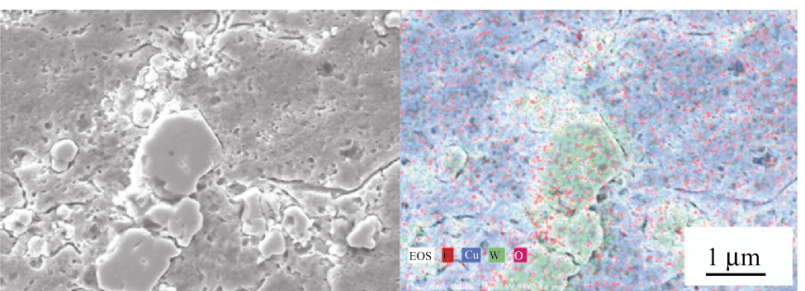

(c) SLD-3 kW

图 6 SLD 复合涂层 EDS 分析

\section{$2.4 \mathrm{WC}$ 的分布与含量}

为了研究各涂层中强化相 $\mathrm{WC}$ 的含量以及分布 情况, 选择各个涂层的中部区域进行背散射电子扫 描, 接着利用 Image $\mathrm{J}$ 软件对图片进行二值化处理 得到 WC 颗粒的像素面积并与整个图片像素面积对 比计算得出涂层中 $\mathrm{WC}$ 颗粒的面积占比, 以此来比 较激光功率对涂层中 $\mathrm{WC}$ 颗粒含量的影响, 结果如 图 7 所示。可以看出, 强化相 WC 颗粒在各涂层中 分布较为均匀, $\mathrm{CS}$ 涂层中的 $\mathrm{WC}$ 颗粒面积占比为

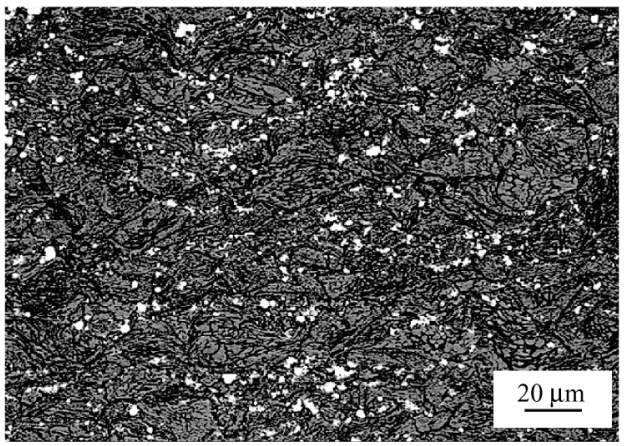

(a) $\mathrm{CS}$

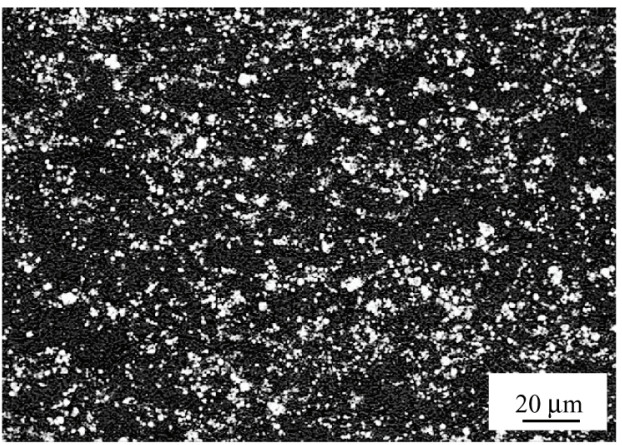

(b) SLD-1 kW

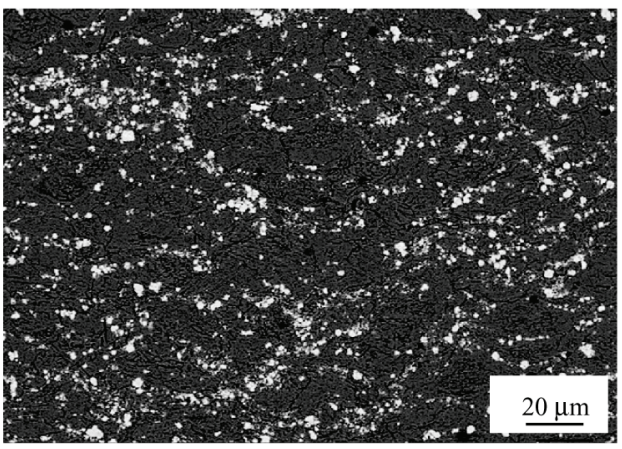

(c) SLD-2 kW

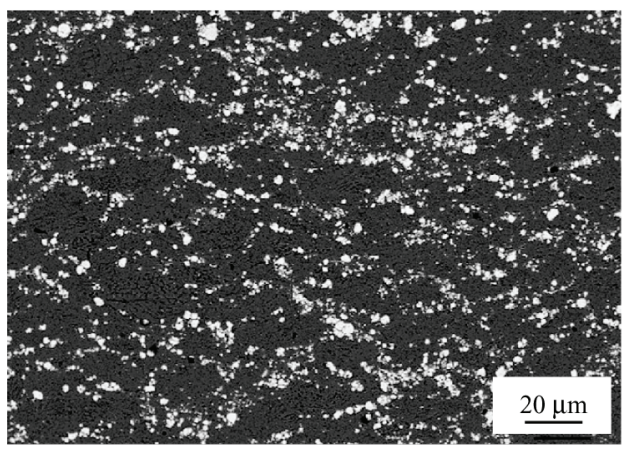

(d) SLD-3 kW

\section{图 7 不同激光功率下涂层中部 WC 颗粒含量分布}

$7.73 \%$; 当激光功率为 $1 \mathrm{~kW}$ 时, $\mathrm{WC}$ 颗粒的面积 占比突增至 $17.22 \%$; 当激光功率为 $2 \mathrm{~kW}$ 时, $\mathrm{WC}$ 颗 粒的面积占比降至 $10.41 \%$ 但仍高于 CS 涂层; 继续 升高激光功率为 $3 \mathrm{~kW}$ 时, WC 颗粒的面积占比增 加至 $12.41 \%$ 。在冷喷涂中, 类似于 $\mathrm{WC}$ 的硬脆的陶 瓷强化相通常不易发生形变, 所以需要具有良好塑 性的粘结相与之结合来形成复合涂层。而在超音速 激光沉积中，由于外加激光辐照对粉末与基材的加 热软化作用, $\mathrm{Cu}$ 粘结相的塑性变形能力大大提高, 从而高速的 $\mathrm{WC}$ 颗粒能更好地和粘结相结合而形成 复合涂层。但在本研究中，复合涂层中的 WC 含量 并没有单调的随着激光功率的增加而增加, SLD-2 $\mathrm{kW}$ 和 SLD-3 $\mathrm{kW}$ 涂层中的 WC 含量相比于 SLD-1 kW 涂层出现了一定的减少, 但均高于 CS 涂层。这种情况表明, 激光辐照的引入所带来的粉 末与基材的软化效应确实有利于 $\mathrm{WC}$ 的沉积，但过 高的激光功率会使得粘结相发生一定程度的氧化, 这对于 $\mathrm{WC}$ 的沉积是不利的。

\section{5 复合涂层的硬度与摩擦磨损性能}

图 8 所示为在不同激光功率下沉积的涂层的显 微硬度。由于 CS 过程中颗粒高速碰撞所带来的加 工硬化效果, $\mathrm{CS}$ 涂层的平均显微硬度达到 $147.4 \mathrm{HV}_{0.2}$ 。SLD-1 $\mathrm{kW}$ 涂层的平均显微硬度达到

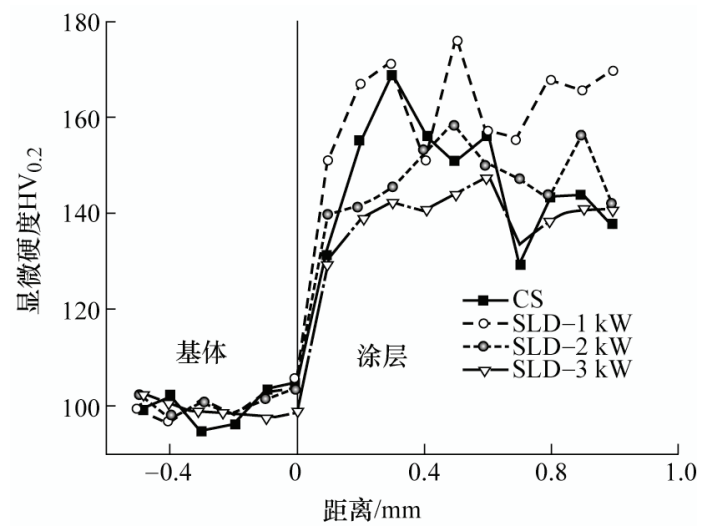

图 8 复合涂层的显微硬度曲线 
了 $161.3 \mathrm{HV}_{0.2}$, 但随着激光功率的增加, 涂层硬度 逐渐降低。SLD-1 kW 涂层的高硬度与其高的 WC 含量有关, 但 SLD-2 kW 与 SLD-3 kW 的 WC 含量 均高于 $\mathrm{CS}$ 涂层但硬度值却低, 这种情况说明了同 步激光辐照对沉积涂层具有软化效果, 这类似于冷 喷涂涂层的退火处理 ${ }^{[23]}$ 。

对纯铜、CS 涂层和 SLD-1 kW 涂层的摩擦磨损 性能进行了对比研究, 摩擦曲线和磨损磨痕及磨损量 对比如图 9 11 所示。可以发现, 纯铜的摩擦因数比 复合涂层高, 这说明在铜基体表面沉积 $\mathrm{WC} / \mathrm{Cu}$ 复合 涂层可以有效地降低纯铜的摩擦因数以及增强耐磨 性能。SLD-1 kW 涂层的摩擦因数比 CS 涂层的摩擦 因数更小且更稳定。在 $60 \mathrm{~min}$ 滑动后, SLD-1 $\mathrm{kW}$ 涂 层的平均摩擦因数约为 0.443 , 而在相同的滑动时间后, CS 涂层的平均摩擦因数约为 0.538 。磨损后的磨痕宽 度分别为 $1238 \mu \mathrm{m} 、 1150 \mu \mathrm{m} 、 1072 \mu \mathrm{m}$, 磨损量分 别为 $4.01 \mathrm{mg} 、 3.01 \mathrm{mg} 、 2.52 \mathrm{mg}$, 这进一步说明

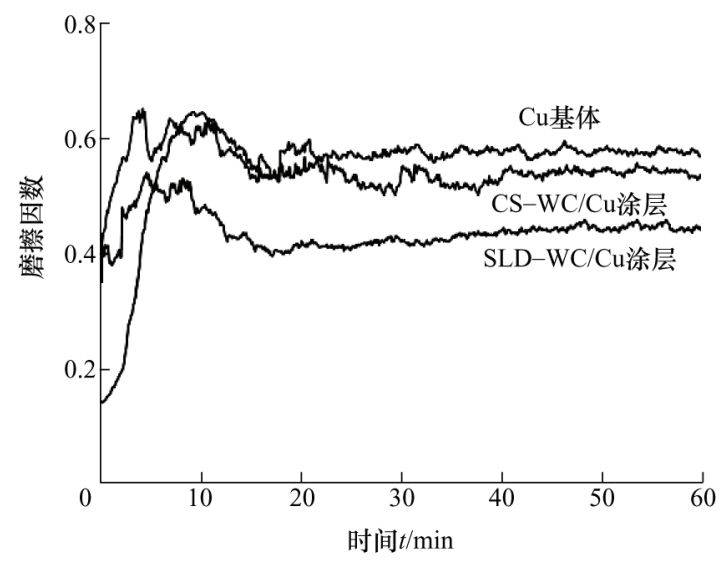

图 9 摩擦因数曲线

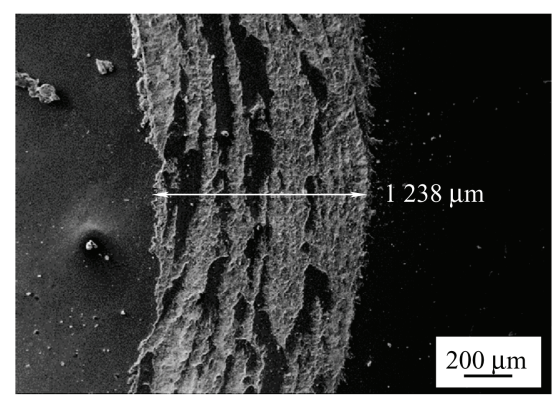

(a) $\mathrm{Cu}$ 基体

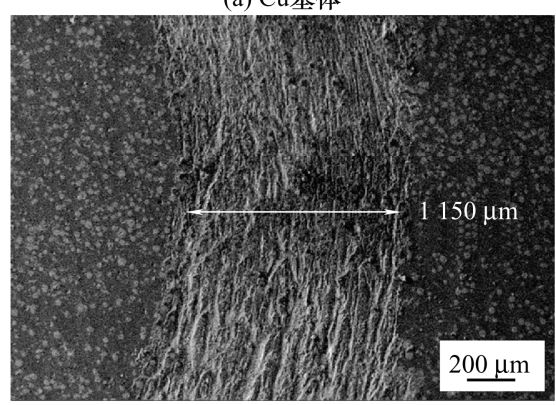

(b) CS涂层

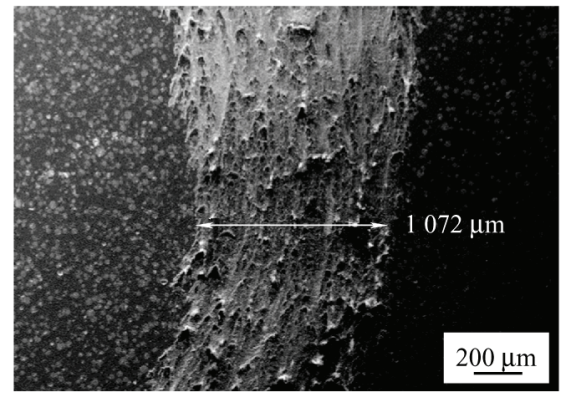

(c) SLD-1 kW涂层

图 10 磨损磨痕

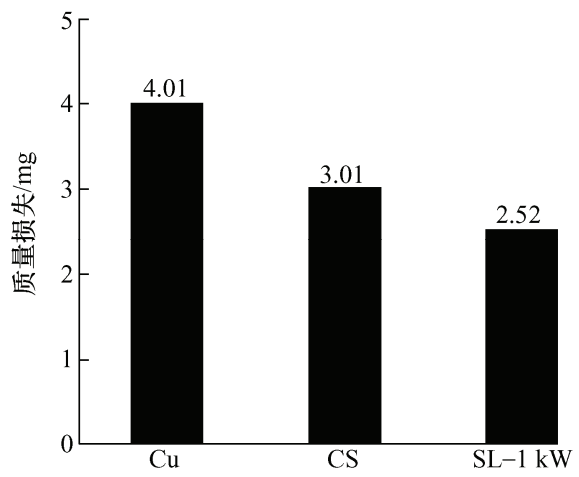

图 11 磨损量

了 WC 的加入改善了纯铜的耐磨性能, 且 SLD 涂层 的耐磨性能优于 CS 涂层。

通过观察磨痕的形态, 可以进一步理解纯铜、 CS 涂层和 SLD-1 $\mathrm{kW}$ 涂层之间的磨损行为的差异。 图 12 为放大 1000 倍下磨痕中心的微观形貌。可以 看出纯铜的磨痕中有较大的剥落区, 磨损机制主要 为粘着磨损; CS 涂层和 SLD-1 $\mathrm{kW}$ 涂层表面有一 些较浅的犁沟, 无大的剥落区, 这是在磨损过程中, 随着粘结相被磨损，有细小的 WC 颗粒从涂层中剥 落, 随着摩擦副的转动对粘结相造成刮伤, 复合涂 层的磨损机制变为粘着磨损和磨粒磨损。 SLD-1 $\mathrm{kW}$ 涂层由于其 $\mathrm{WC}$ 含量高, 因此显示出较 低的摩擦因数; 强化相 $\mathrm{WC}$ 和粘结相 $\mathrm{Cu}$ 结合比 $\mathrm{CS}$ 涂层更好, 使得 WC 脱落导致磨粒磨损的概率要小, 从而磨损后的磨痕较浅，表现出更好的耐磨性。

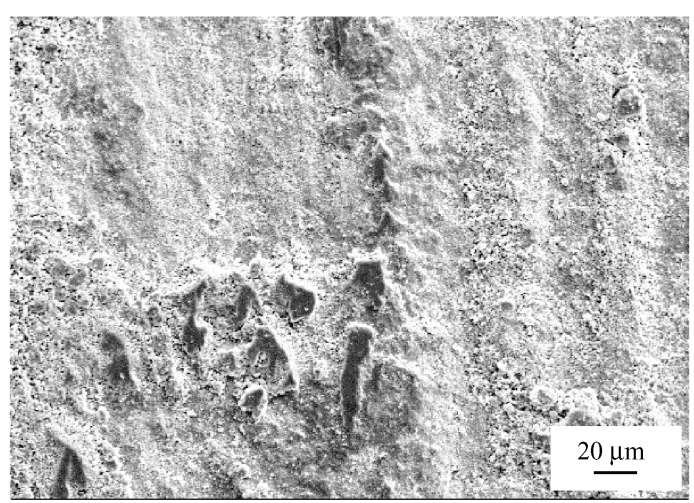

(a) $\mathrm{Cu}$ 基体 


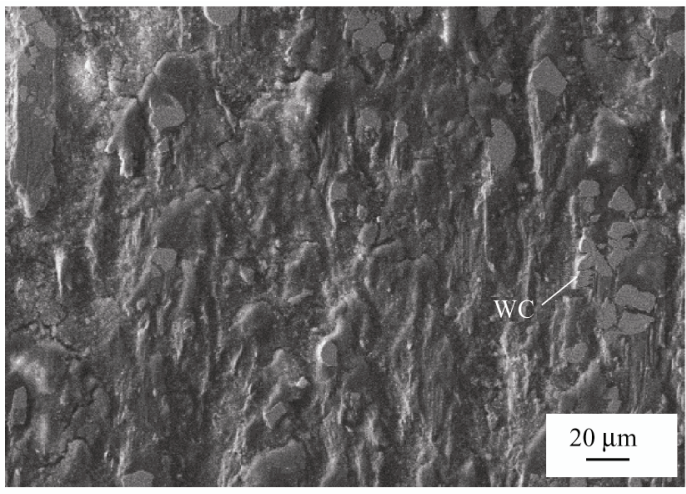

(b) CS 涂层

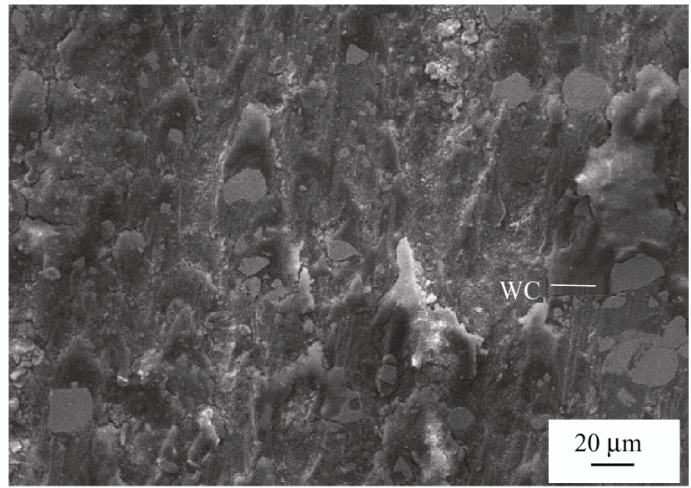

(c) SLD-1 kW涂层

图 12 磨损磨痕中心

\section{3 结论}

采用 SLD 与 $\mathrm{CS}$ 在纯铜表面制备了 $\mathrm{WC} / \mathrm{Cu}$ 复 合涂层并对沉积层的微观结构、相组成、显微硬度 和摩擦磨损性能进行了对比分析, 得到以下结论。

(1) 在激光辐照的软化作用下, SLD 涂层的 厚度均高于 $\mathrm{CS}$ 涂层, 且厚度随着激光功率的增 加而逐渐增加。CS 涂层与基体的结合处存在由 于结合差而产生的缝隙, SLD 涂层表现出更好的 界面结合情况。

(2) 由于热输入相对较低, SLD 涂层可以保 持与原始粉末相同的物相成分。但是, 对于在高 激光功率下制备的样品, 可以检测到轻微的氧 化, 这会对颗粒沉积有一定的不利影响。强化相 $\mathrm{WC}$ 颗粒较为均匀分散在复合涂层中, SLD-1 kW 涂层比其他涂层具有更高的 $\mathrm{WC}$ 颗粒含量以及更 高的显微硬度。

(3) 铜基体的磨损机制为粘着磨损, 复合涂层 的磨损机制主要为粘着磨损和磨粒磨损。SLD 涂层 的摩擦因数小于 CS 涂层和铜基体, 且磨痕宽度更 小, 磨损量更少, 显示出更好的耐磨性能。因此在 纯铜表面利用 SLD 制备 $\mathrm{WC} / \mathrm{Cu}$ 涂层能够改善其硬 度与耐磨性能。

\section{参 考 文 献}

[1] 张跃飞, 苏永安, 陈飞. 高强度高导铜基材料研究进展 [J]. 国外金属热处理，2001，22：6-9.

ZHANG Yuefei, SU Yongan, CHEN Fei. Research on high strength and high conductivity copper-based materials[J]. Heat Treatment of Metals Abroad, 2001，22: 6-9.

[2] 刘涛, 䣓剑, 凌国平, 等. 颗粒增强铜基复合材料研究 进展 [J]. 材料导报, 2004，18: 53-55.

LIU Tao, LI Jian, LING Guoping, et al. Progress in studies of particle reinforced Cu-matrix composites[J]. Materials Review, 2004, 18: 53-55.

[3] IBRAHIM A E A M, KIM H S. Effect of the fabrication method on the wear properties of copper silicon carbide composites[J]. Tribology International, 2018, 128: $140-154$

[4] WANG Y, CHEN M, ZHOU F, et al. High tensile ductility in a nanostructured metal[J]. Nature, 2002, 419(6910): 912-915.

[5] 袁俊瑞, 徐佳, 周振宇, 等. 滚压诱导纯铜表面梯度纳米 结构磨损行为研究[J]. 机械工程学报, 2017, 53(24): 49-54. YUAN Junrui，XU Jia，ZHOU Zhenyu，et al. Study on wear behavior of gradient nanocrystalline structure on pure copper surface induced by burnishing[]]. Journal of Mechanical Engineering, 2017, 53(24): 49-54.

[6] KANG H K. Microstructure and electrical conductivity of high volume $\mathrm{Al}_{2} \mathrm{O}_{3}$-reinforced copper matrix composites produced by plasma spray[J]. Surface \& Coatings Technology, 2005, 190(2-3): 448-452.

[7] 刘德宝, 崔春翔, 马叙. 氮化铝颗粒表面镀铜及其增强铜 基复合材料 [J]. 兵器材料科学与工程, 2005, 28(2): 8-11. LIU Debao, CUI Chunxiang, MA Xu. Surface coating copper on AIN particle and AIN particle-reinforced copper matrix composites[]]. Ordnance Material Science and Engineering, 2005, 28(2): 8-11.

[8] DESHPANDE P K, LI J H, LIN R Y. Infrared processed $\mathrm{Cu}$ composites reinforced with WC particles[J]. Materials Science \& Engineering A (structural materials : properties, microstructure and processing), 2006, 429(1-2): 58-65.

[9] 杨贵荣, 宋文明, 郝远, 等. 铜基表面铸渗法 $\mathrm{Ni} / \mathrm{Al} 2 \mathrm{O} 3$ 复 合渗层的组织和性能 []]. 腐蚀与防护, 2007, 28(6): 275-278. YANG Guirong, SONG Wenming, HAO Yuan, et al. Microstructure and properties of $\mathrm{Ni} / \mathrm{Al} 2 \mathrm{O} 3$ composite infiltrated layer on copper surface[J]. Corrosion \& Protection, 
$2007,28(6): 275-278$.

[10] 曾爱香, 唐绍装. 金属基陶瓷涂层的制备、应用及 发展 $[\mathrm{J}]$. 陶瓷研究, 1998(4): 7-10.

ZENG Aixiang, TANG Shaoqiu. Preparation, application and development of metal-based ceramic coatings[J]. Ceramic Studies Journal, 1998(4): 7-10.

[11] BRAY M, COCKBURN A, O'NEILL W. The laser-assisted cold spray process and deposit characterization[J]. Surface and Coatings Technology, 2009, 203(19): 2851-2857.

[12] YAO J H, YANG L J, LI B, et al. Beneficial effects of laser irradiation on the deposition process of diamond/Ni60 composite coating with cold spray[J]. Applied Surface Science, 2015, 330: 300-308.

[13] LI B, YAO J H, ZHANG Q L, et al. Microstructure and tribological performance of tungsten carbide reinforced stainless steel composite coatings by supersonic laser deposition[J]. Surface and Coatings Technology, 2015, 275: $58-68$.

[14] 姚建华, 吴丽娟, 李波, 等. 超音速激光沉积技术: 研 究现状及发展趋势 $[J]$. 中国激光，2019，46(3)：9-19. YAO Jianhua, WU Lijuan, LI Bo, et al. Supersonic laser deposition: The state of the art and future perspective[J]. Chinese Journal of Lasers， 2019， 46(3): 9-19.

[15] KULMALA M, VUORISTO P. Influence of process conditions in laser-assisted low-pressure cold spraying $[\mathrm{J}]$. Surface \& Coatings Technology, 2008, 202(18) : 4503-4508.

[16] LI B, YANG L J, LI Z H, et al. Beneficial effects of synchronous laser irradiation on the characteristics of cold-sprayed copper coatings[J]. Journal of Thermal Spray Technology, 2015, 24(5): 836-847.

[17] 骆芳, 赵兵, 姚建华. 激光辅助冷喷涂 $\mathrm{Cu}$ 颗粒撞击 45 钢的数值模拟 $[\mathrm{J}]$. 兵工学报, $2015,36(11): 2157-2163$. LUO Fang, ZHAO Bing, YAO Jianhua. Numerical simulation of $\mathrm{Cu}$ particles impacting on medium steel in laser-assisted cold spraying[J]. Acta Armamentarii, 2015,
36(11): 2157-2163.

[18］吴丽娟，汪伟林，李波，等. 金刚石粒径及含量对超音 速激光沉积金刚石 $/ \mathrm{Cu}$ 复合涂层微观结构及性能的影 响 [J]. 表面技术，2019，48(2)：50-56.

WU Lijuan, WANG Weilin, LI Bo, et al. Influence of diamond particle size and content on the microstructure and properties of diamond/ $\mathrm{Cu}$ composite coating prepared by supersonic laser deposition[J]. Surface Technology, 2019, 48(2): 50-56.

[19] ASSADI H, GARTNER F, STOLTENHOFF T, et al. Bonding mechanism in cold gas spraying[J]. Acta Materialia, 2003, 51(15): 4379-4394.

[20] LI B, JIN Y, YAO J H, et al. Influence of laser irradiation on deposition characteristics of cold sprayed Stellite 6 coatings[J]. Optics \& Laser Technology, 2018, 100: 27-39.

[21］李波, 李鹏辉, 张群莉, 等. 超音速激光沉积 WC/SS316L 复合涂层微观结构及磨损性能研究 [J]. 电加工与模具, 2016(1): 35-39.

LI Bo, LI Penghui, ZHANG Qunli, et al. Study on microstructure and wear-resistant properties of $\mathrm{WC} / \mathrm{SS} 316 \mathrm{~L}$ composite coatings prepared by supersonic laser deposition[J]. Electromachining \& Mould, 2016(1): 35-39.

[22] 赵兵. 超音速沉积 Stellite 6 颗粒与激光复合工艺的数 值模拟研究[D]. 杭州: 浙江工业大学, 2015.

ZHAO Bing. Numerical simulation on supersonic deposition stellite 6 particles using the laser hybrid technology[D]. Hangzhou: Zhejiang University of Technology, 2015.

[23] MENG X M, ZHANG J B, HAN W, et al. Influence of annealing treatment on the microstructure and mechanical performance of cold sprayed 304 stainless steel coating $[\mathrm{J}]$. Applied Surface Science, 2011， 258(2): 700-704.

作者简介: 黄煊杰, 男, 1995 年出生, 硕士研究生。主要研究方向为激 光复合表面改性技术。

E-mail: xuanjiehuang@foxmail.com

姚建华(通信作者), 男, 1965 年出生, 博士, 教授, 博士研究生导师。 主要研究方向为激光复合制造。

E-mail: laser@zjut.edu.cn 